\title{
Fundus Eye Images Classification for Diabetic Retinopathy Detection Using Very Deep Convolutional Neural Network
}

\author{
Ítalo Rodrigues Gama*, Alessandra Martins Coelho *, Matheus de Freitas Oliveira Baffa ${ }^{\dagger}$ \\ ${ }^{*}$ Instituto Federal de Educação, Ciência e Tecnologia do Sudeste de Minas Gerais (IF Sudeste MG) \\ Rio Pomba, MG, Brasil \\ †Universidade de São Paulo (USP) \\ Ribeirão Preto, Brasil \\ Email: itrgama@gmail.com, alessandra.coelho@ifsudestemg.edu.br, mfreitas826@gmail.com
}

\begin{abstract}
Diabetic retinopathy is an anomaly responsible for causing microvascular and macrovascular damage to the retina and occurs as a consequence of the worsening of diabetes. According to the World Health Organization (WHO), diabetic retinopathy is the most common cause of avoidable blindness in patients with diabetes worldwide. Early detection is important for the efficiency of treatments. Fundus Eye Image can be used to identify early disease development and monitor the patient's clinical condition. The diagnostic process using this type of image may require some expertise from the ophthalmologist since not all retina anomalies are clearly visible. Thus, this paper proposes the development of a classification method based on Convolutional Neural Networks, but highly dense and deeper. The proposed method obtained a total of $92 \% \mathrm{AUC}$ in the given experiments.
\end{abstract}

\section{INTRODUÇÃo}

Considerada como uma epidemia mundial, a diabetes é um grupo de distúrbios metabólicos que se caracteriza pela hiperglicemia causada pela ação ou secreção anormal da insulina, que leva a alterações no metabolismo de carboidratos, lipídios e proteínas [4]. Segundo a Secretaria de Saúde do Estado do Paraná, esses distúrbios, ao longo do tempo, comprometem a função e a estrutura vascular de diferentes órgãos, como o coração, os rins e os olhos [5].

A diabetes pode gerar danos significativos, tanto econômicos quanto sociais. As estatísticas indicam que o número de casos em todo mundo atualmente chega a 463 milhões, com expectativa de alcançar 700 milhões de pessoas em 2045. De acordo com dados da Federação Internacional de Diabetes, em 2019 a diabetes causou 4,2 milhões de mortes e estima-se que foi responsável por 760 bilhões de dólares gastos com saúde, cerca de 10\% do gasto global [2].

A retinopatia diabética é uma das principais complicações relacionadas a diabetes e a principal causa da perda de visão em pessoas com idade entre 20 e 74 anos [13]. Ela se caracteriza por lesões nos capilares da retina, causando inicialmente vazamentos que levam a inchaços e hemorragias. Mais tarde, há a proliferação de vasos sanguíneos no interior do olho, descolamento de retina e glaucoma [9]. Após 20 anos de doença, cerca de $75 \%$ dos diabéticos apresentam algum grau de retinopatia [14].
$\mathrm{O}$ exame mais fundamental para que o oftalmologista possa avaliar alterações oculares, inclusive em pacientes diabéticos, é o exame de fundo de olho, ou fundoscopia. Neste exame, procura-se avaliar as estruturas do fundo de olho, dando atenção ao nervo óptico, aos vasos sanguíneos e a mácula, região central da retina [3].

Caso a retinopatia diabética seja detectada, o acompanhamento deverá ser periódico, conforme a intensidade da doença no olho e o quadro clínico geral do paciente. Quando o diagnóstico é realizado em tempo adequado e o tratamento realizado corretamente, complicações graves podem ser retardadas ou impedidas por completo, reduzindo o risco de cegueira dessa anomalia a menos de 5\% [10].

A partir dos anos 80, tornou-se habitual realizar diagnósticos auxiliado por computador (CAD - Computer-aided +). Esses sistemas enfatizam áreas de importância e apresentam possíveis diagnósticos para alguma anomalia detectada. Geralmente, um sistema CAD é composto por um algoritmo de pré-processamento da base de dados, um algoritmo de classificação e uma interface gráfica para manuseio da aplicação [15].

Os sistemas de diagnóstico auxiliado por computador têm como objetivo auxiliar o médico especialista melhorar a consistência da interpretação da imagem utilizando a resposta do computador como referência. Dessa forma, uma dupla analise realizada pelo computador em conjunto com médico especialista pode melhorar a eficiência do diagnóstico [11].

Ao longo dos anos foram desenvolvidos diferentes métodos de classificação para utilização em imagens oftalmológicas. Destas, destaca-se (i) o método de morfologia matemática para detectar exsudatos em imagens de fundo de olho de pacientes diabéticos [25], (ii) o emprego de técnicas de segmentação de bordas para detectar vasos sanguíneos e classificar imagens com retinopatia [26], (iii) a aplicação da transformada de wavelet para classificação da retinopatia diabética [20] e (iv) a utilização do aprendizado profundo, através da Rede Neural Convolutiva, para extrair características das imagens de fundo de olho em conjunto de um algoritmo de árvore de decisão para realizar a identificação da retinopatia diabética [12]. 
O presente trabalho tem como objetivo desenvolver um método de classificação para auxiliar médicos oftalmologistas na detecção da retinopatia diabética, em qualquer grau. Para isto, será empregado um algoritmo de aprendizado de máquina baseado na abordagem do aprendizado profundo, para encontrar padrões nos exames de fundo de olho e classificá-los de acordo com o estado de saúde do paciente, entre doente e saudável.

$\mathrm{O}$ presente trabalho contribui à comunidade acadêmica com os seguintes achados:

- o desenvolvimento de um novo método para a padronização de bases de imagens de fundo de retina

- o desenvolvimento de uma arquitetura de Rede Neural Convolucional baseada nos modelos existentes de Redes Altamente Profundas

- a avaliação das arquiteturas atuais de Redes Convolutivas Altamente Profundas em bases não padronizadas

- o desenvolvimento de um método que integra o PréProcessamento das Imagens e as Redes Neurais Convolucionais para realizar a classificação de imagens de fundo de retina

- uma avaliação do método proposto com demais trabalhos da literatura

O artigo está organizado como se segue. Na seção II é realizado um estudo do atual estado da arte em classificação de exames de fundo de olho para detectar a retinopatia diabética. Em seguida, nas seções III e IV são descritas as metodologias de pré-processamento e da classificação da base de dados, contribuição deste trabalho. Os experimentos realizados e os resultados obtidos são apresentados na seção V e na seção VI, encontra-se a conclusão do presente trabalho.

\section{TRABAlhos CoRrelatos}

Os trabalhos a seguir tratam de metodologias para a detecção da retinopatia diabética.

\section{A. Gargeya and Leng [12]}

Neste trabalho foi desenvolvido um algoritmo de classificação de imagens de fundo de olho utilizando Redes Neurais Profundas. A base de dados EyePACS utilizada neste trabalho, possuía 75 mil imagens e eram oriundas de um projeto envolvendo especialistas em oftalmologia, que coletaram e classificaram manualmente os exames.

Devido as imagens terem sido obtidas utilizando diferentes protocolos de aquisição, a base de dados era bem heterogênea. Assim, fez-se necessário realizar uma etapa de pré-processamento para padronizar essas imagens. Para o treinamento do método de classificação, utilizou-se uma rede neural convolucional, devido à sua ampla aplicabilidade e robustez em tarefas de reconhecimento em bases de grande quantidade.

Utilizando validação cruzada, esse modelo alcançou uma área abaixo da curva ROC (Area Under the Curve - AUC) de 0,97 , com sensibilidade e especificidade de $94 \%$ e de $98 \%$ em dados locais. Para validação externa foram utilizados os bancos de dados Messidor [7] e E-Ophtha [6], alcançando uma pontuação de 0,94 e 0,95 , respectivamente.

\section{B. Pratt et al. [18]}

O modelo proposto utilizou 80 mil imagens de fundo de olho adquiridas da base de dados Kaggle, composta por imagens de aproximadamente seis milhões de pixels cada. Para o treinamento utilizou-se a rede neural convolucional (CNN) processada em uma GPU NVDIA K40c (2880 núcleos CUDA).

O conjunto de dados de imagens de fundo de olho se refere a pacientes de diferentes etnias, faixas etárias e níveis variados de iluminação, fato que afeta os valores de intensidade de pixel nas imagens. Para combater variações desnecessárias o autor aplicou a normalização de cores usando o pacote OpenCV. As imagens foram redimensionadas para $512 \times 512$ pixels devido o alto custo de processamento.

Foram separadas cinco mil imagens para validação da CNN. A execução das imagens levou cerca de 188 segundos e o resultado final da rede treinada foi $95 \%$ de especificidade, $75 \%$ de acurácia e $30 \%$ de sensibilidade.

\section{Sinthanayothin et al. [22]}

Neste trabalho foi proposto uma metodologia para análise de imagens de fundo de olho caracterizando a retinopatia diabética não-proliferativa. Foram utilizadas 112 imagens digitais de fundo de olho, capturadas usando uma câmera não midriática, de pacientes atendidos em um centro de triagem. Foi necessário um pré-processamento das imagens para combater o desbalanceamento de contraste, uma vez que em uma mesma imagem os níveis de contraste no centro eram superiores aos níveis de contraste nas bordas. A equalização de histograma adaptativa foi aplicada para minimizar o desbalanceamento, produzindo assim uniformidade na imagem.

$\mathrm{O}$ algoritmo de reconhecimento de exsudatos foi aplicado em 30 imagens de fundo de olho, das quais 21 continham exsudatos e nove estavam sem indicadores. A sensibilidade e especificidade para detecção de exsudato foram $88,5 \%$ e $99,7 \%$, respectivamente.

\section{Verma et al. [23]}

Neste trabalho foi proposto um método para classificar os diferentes estágios da retinopatia diabética baseado na quantificação de vasos sanguíneos e hemorragia presente na retina. Foram utilizadas 65 imagens de fundo de olho, sendo, 30 imagens de retina normal, 23 com retinopatia diabética moderada e 12 com grave.

Primeiramente é realizada a segmentação através da diferença de contraste entre os vasos sanguíneos e o fundo. Logo após são utilizadas técnicas de análise de densidade e caixa delimitadora para detectar os exames que possuíam hemorragia. Por último a classificação dos diferentes estágios da anomalia foi realizado através da técnica de Florestas Aleatórias com base na área e perímetro dos vasos sanguíneos e nas hemorragias.

O método proposto classificou com precisão de $90 \%$ os casos normais, enquanto moderada e grave $87,5 \%$. 


\section{Pré-Processamento da Base de Dados}

O treinamento e a avaliação do método de classificação foi realizada utilizando duas bases de dados, o iDRIB [17] e o Messidor [7]. Ambas as bases podem ser acessadas e baixadas gratuitamente pela internet.

A base de dados iDRIB é composta por 516 imagens divididas em dois grupos: (i) retinas com sinais de retinopatia diabética e; (ii) retinas saudáveis sem sinais da doença. Todas as imagens foram classificadas manualmente pelos oftalmologistas de acordo com a presença ou não de anomalias. Em (a) na (Fig. 1) estão exemplos de imagens de fundo de olho obtidas através da base de dados citada anteriormente.

A Messidor por sua vez, é composta por 1200 imagens de fundo de olho coletados por três especialistas em oftalmologia. Destas, 800 imagens foram obtidas utilizando dilatação da pupila e 400 sem dilatação. As imagens nesta base de dados são disponibilizadas junto de um diagnóstico realizado pelo médico responsável pela coleta. Em (b) na (Fig. 1) estão exemplos de imagens de fundo de olho obtidas através da base de dados citada acima, é visível a diferença de padronização entre as bases de dados (a) e (b).

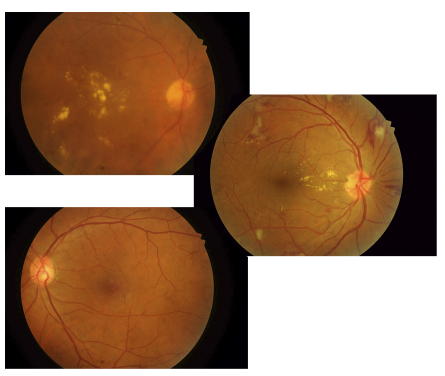

(a)

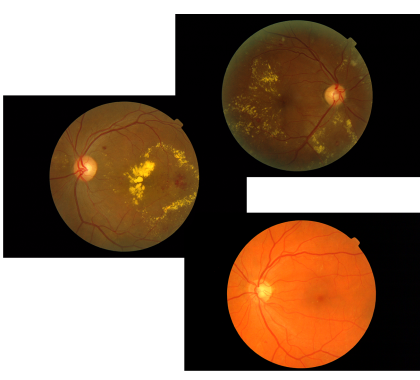

(b)
Fig. 1. Exemplos de imagens de fundo de olho presentes nas bases de dados. Em (a), a base de dados iDRIB e em (b), a base de dados Messidor. Fonte: autor.

Ambas as bases de dados possuem amostras de retina de pacientes doentes e saudáveis. Os pacientes doentes apresentam esperadas anomalias que permitem diferenciar visualmente ambas as classes. Das anomalias presentes nas imagens doentes, destacam-se a presença de exsudatos duros e algodonosos, e de hemorragias e microaneurismas.

Além desse padrão visível, as imagens de fundo de olho são sensíveis à idade do paciente (Fig. 2). Essas podem se apresentar com um aspecto borrado, para pacientes idosos, ou com reflexos, e estruturas bem definidas e saltadas em casos de pacientes mais jovens.

Outro padrão visual presente nas imagens de fundo de olho tem relação com a raça do paciente (Fig. 3). Pacientes de pele morena ou negra tendem a possuir uma retina com pigmentação mais escura, devido a alta presença de melanina nas células que compõe esta estrutura ocular. Os pacientes de pele mais clara possuem menor pigmentação de melanina na retina que, por sua vez, se apresenta com a coloração alaranjada e/ou avermelhada.
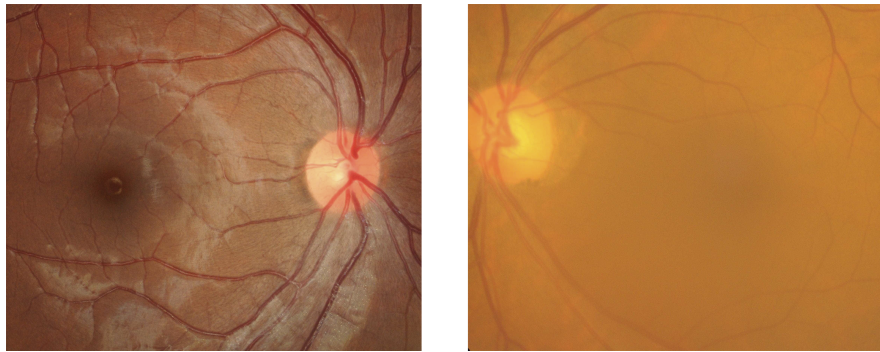

Fig. 2. A visualização da retina de um paciente jovem, com reflexo e estruturas bem definidas, e um paciente idoso com aspecto borrado e estruturas mais suaves. Fonte: autor.

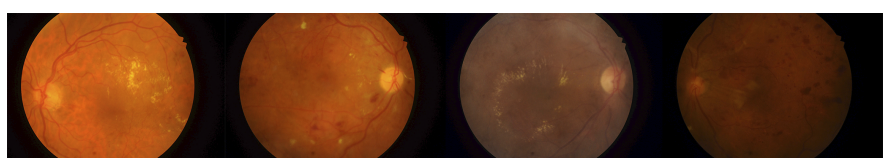

Fig. 3. Os diferentes níveis de melanina na retina, de acordo com a raça do paciente. Fonte: autor.

Tais padrões visuais que não caracterizam pacientes doentes ou saudáveis dificultam a construção de classificadores de alta eficiência. Um estudo preliminar realizado neste trabalho, utilizando diferentes arquiteturas de Redes Neurais Convolutivas, mostrou que as imagens apresentam baixas taxas de acerto, quando utilizadas sem qualquer tratamento.

A Tabela I contém a acurácia média de validação em três diferentes arquiteturas de Redes Neurais Convolutivas. Para estes testes não foram utilizadas técnicas de processamento ou melhoramento das imagens. Observa-se que, mesmo arquiteturas sofisticadas e recentes, como a Visual Geometric Group - VGG e a Residual Neural Network - ResNet não obtiveram bons resultados quando aplicadas ao problema de classificação binária de exames de fundo de olho. Então, fez-se necessário adicionar uma etapa de pré-processamento antes da construção de um classificador. O objetivo desta etapa foi padronizar a distribuição de cor entre os exames e destacar estruturas da retina que pudessem ser importantes para diferenciar os padrões saudáveis dos demais padrões relacionados a doenças.

TABELA I

COMPARATIVO ENTRE A ACURÁCIA DE TRÊS DIFERENTES ARQUITETURAS DE REDES NEURAIS CONVOLUTIVAS SEM O PRÉ-PROCESSAMENTO NA BASE DE DADOS. FONTE AUTOR.

\begin{tabular}{|l|r|l|}
\hline Arquitetura & Acurácia Média & Imagens Processadas? \\
\hline LeNet & $55 \%$ & Não \\
\hline VGG & $45 \%$ & Não \\
\hline ResNet & $51 \%$ & Não \\
\hline
\end{tabular}

A construção desta etapa auxiliou na criação de um método de classificação generalizado. Desta forma, tornou-se possível aplicar a presente metodologia à diferentes bases de dados, com imagens obtidas seguindo diferentes protocolos de aquisição. 


\section{A. Visão geral do método de padronização}

A cor da retina não é uma característica importante para dizer se um paciente está doente ou saudável. Desta forma, neutralizar os efeitos que esta característica pode causar no classificador é a primeira etapa do método proposto, o qual utiliza apenas um canal de cor no formato de uma imagem em tons de cinza.

Em seguida, a imagem em tons de cinza passa por um processo de equalização do histograma e é convertida em negativo. Esta etapa auxilia na construção de imagens mais homogêneas em relação à distribuição da cor e em relação às estruturas que fazem parte da retina.

Por fim, realiza-se um recorte do interior da retina, removendo toda informação de fundo desnecessária. Esta ação também padroniza o tamanho e a informação contida nas imagens vindas de diferentes fontes de dados. Na Figura 4, um fluxograma do método de padronização desenvolvido neste trabalho é apresentado.

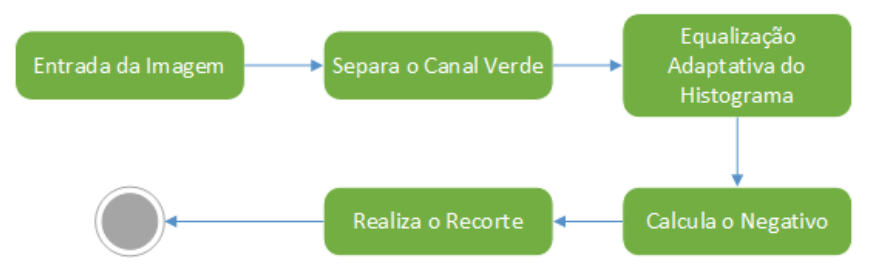

Fig. 4. Fluxograma do método de padronização das imagens de fundo de olho. Fonte: autor.

\section{B. Visão detalhada do método de padronização}

Nesta seção iremos detalhar as etapas que compõem o processo de padronização das bases de dados.

1) Extração do Canal Verde: A fim de reduzir os efeitos da cor da retina no processo de busca por padrões, foi proposta inicialmente a conversão da imagem para uma escala de tons de cinza. Entretanto, a partir de um estudo dos canais de cores, notou-se que o canal de cor verde apresentava visualmente informações mais ricas e detalhadas acerca da retina, das suas estruturas adjacentes e dos sinais patológicos. Desta forma, em vez de de empregar a tradicional conversão do espaço de cor RGB para tons de cinza, os canais de cores da imagem foram separados e a extração do canal verde foi utilizado como método de conversão da imagem colorida para tons de cinza.

A Figura 5 possui um exemplo de conversão de um exame em tons de cinza utilizando tanto o método tradicional quanto a separação dos canais de cores. Observa-se que, utilizando o canal verde é possível obter mais detalhes sobre as estruturas da retina, como o disco óptico e os vasos sanguíneos, além de destacar as anomalias presentes, como os exsudatos duros, algodonosos e as hemorragias.

2) Equalização Adaptativa de Histograma: Após a etapa de conversão da imagem colorida para escala de tons de cinza, as imagens continuaram se apresentando heterogêneas, dado ao fato de que algumas imagens eram mais escuras e outras mais claras. Desta forma, as imagens foram submetidas a um processo de equalização do histograma.

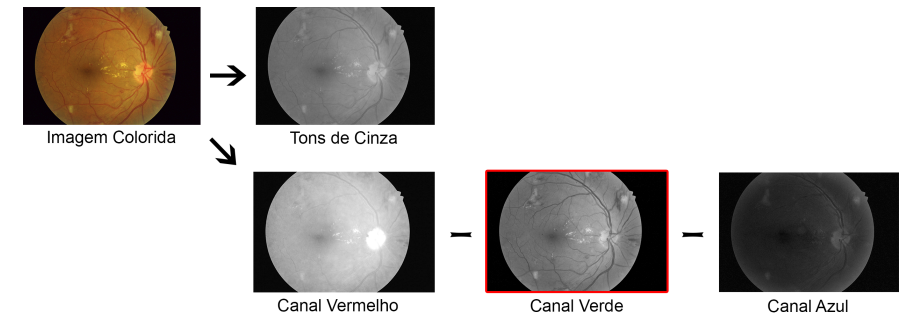

Fig. 5. Conversão da imagem colorida em tons de cinza extraindo canais de cor. Fonte: autor.

Durante o processo de equalização de histograma das imagens, notou-se o desenvolvimento de regiões, hora muito escuras, hora muito claras. A equalização resolvia o problema de homogeneização da base de dados, porém diminuía a qualidade da informação contida nas imagens. Para contornar tal problema, foi utilizado o método de Equalização de Histograma Adaptativa Limitado pelo Contraste (ContrastLimited Adaptive Histogram Equalization - CLAHE) [28]. Este método realiza a equalização baseada em pequenas regiões da imagem, além de limitar o contraste local, evitando que determinadas regiões fiquem ou claras ou escuras em demasia.

3) Cálculo do negativo: Outra medida para auxiliar na homogeneização da base de dados foi a conversão das imagens em seu negativo. Desta forma, destacou-se as anomalias presentes na retina, deixando-as mais escuras. Já as estruturas da retina, como a mácula e os vasos sanguíneos, ficaram de coloração mais clara e menos evidentes.

4) Recorte da região de interesse: Embora ambas as bases de dados possuam a mesma região da retina, as imagens possuem diferentes recortes. Por exemplo, na base de dados iDRIB as imagens são ampliadas e cortadas na região inferior e superior, enquanto na base Messidor as imagens são menores, porém sem cortes. Isso ocorre porque ambas seguem padrões distintos de aquisição de imagens. Assim, para gerar uma base de dados homogênea, foi proposta uma etapa de recorte automático da região interior-central da retina, onde geralmente ocorre a presença das anomalias que permitem a classificação das imagens. Após o recorte todas as imagens ficaram com a resolução de 1100 pixels por 800 pixels.

A Figura 6 possui uma sequência que ilustra os passos da padronização. Observa-se que, independente da base de dados, as imagens finalizam de forma similar e com estruturas e anomalias de fácil identificação.

\section{Desenvolvimento da Rede Neural Convolucional Altamente Profunda}

As metodologias tradicionais de Visão Computacional geralmente são divididas em três etapas, sendo elas (i) o préprocessamento da base de dados, com utilização de técnicas de Processamento de Imagens para realçar características ou até mesmo remover ruídos; (ii) a Análise de Imagens, através da extração de características e representação dessas imagens em um vetor descritor e; (iii) a aplicação de algoritmos de Aprendizado de Máquina para reconhecimento de padrões. 

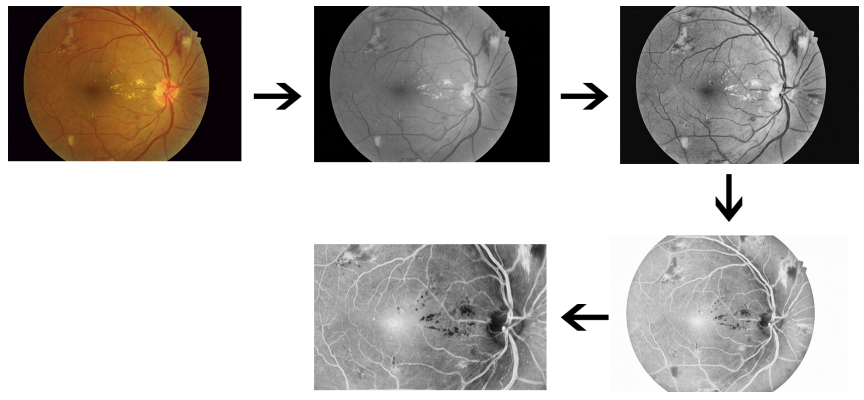

Fig. 6. Esquema visual de padronização da base de dados. Fonte: autor.

Atualmente, é comum ver trabalhos utilizando Redes Neurais Artificiais (Artificial Neural Network - ANN) para o reconhecimento de padrões em imagens médicas [19], [16], [8]. De modo geral, este algoritmo, combinado com uma extração de características efetiva, obtém resultados melhores que outras técnicas de Aprendizado de Máquina, como as Máquinas de Vetor Suporte (Support Vector Machine - SVM) ou as Árvores de Decisão.

Devido ao avanço nas tecnologias de processamento massivo paralelo utilizando placas gráficas (Graphics Processing Unit - GPU), abordagens de Redes Neurais Artificiais, como o Aprendizado Profundo, se fizeram possíveis. Com a alta disponibilidade de dados e poder de processamento, as Redes Neurais Profundas podem detectar padrões complexos através da associação de padrões menores combinados em suas diversas estruturas internas da Rede Neural.

A Rede Neural Convolutiva (Convolutional Neural Network - CNN) é um tipo de arquitetura de Rede Neural Artificial desenvolvida segundo a abordagem das Redes Profundas. Este tipo de rede é capaz de receber uma imagem, atribuir um peso aos diversos objetos e elementos que a compõe, e detectar padrões [1].

Diferente das metodologias tradicionais de Visão Computacional, a CNN reconhece padrões em uma imagem utilizando características extraídas pelas camadas de convolução. A combinação da ordem e da quantidade dessas camadas compreende-se como sub arquiteturas de CNN.

Neste trabalho foi desenvolvida uma sub-arquitetura de CNN baseada na abordagem das Redes Convolutivas Altamente Profundas (Very Deep Convolutional Neural Network VD-CNN). Uma característica dessas redes é a possibilidade de encontrar padrões mais complexos e mais sensíveis. É comprovado que esta metodologia é capaz de superar a performance das Redes Convolutivas Tradicionais ou que seguem o modelo de LeNet [21]. Uma comparação da arquitetura tradicional da CNN com a CNN Altamente Profunda pode ser vista na Figura 7.

A arquitetura de rede neural utilizada neste trabalho (Fig.8) foi desenvolvida baseada na sequência de extração de características da LeNet, contendo mais profundidade baseada na VGG. Nesta arquitetura foram utilizados três grupos de convolução, cada um possuindo, respectivamente, duas camadas de convolução 2D, de 64, 128 e 256 filtros de tamanho

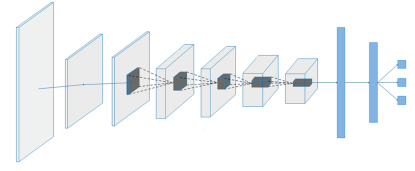

(a)

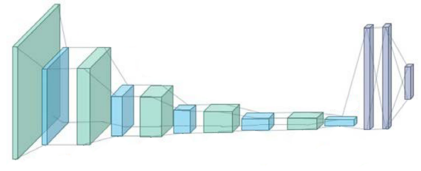

(b)
Fig. 7. Diferentes sub arquiteturas de CNN. Em (a) o modelo de LeNet, em que cada camada de convolução antecede uma camada de Pooling. Em (b) o modelo da VGG, no qual existem mais camadas de Convolução antecedendo as camadas de Pooling. Fonte: [24], [27], adaptado.

3x3, seguidas por uma camada de MaxPooling de tamanho $2 \times 2$. Por fim, as características são enviadas para uma Rede Neural Totalmente Profunda (Fully Connected Neural Network - FCNN) de quatro camadas de 4096 neurônios cada. Nas camadas internas da rede neural foram utilizadas a função de ativação ReLU (Rectifier Linear Unit) em conjunto com o algoritmo de otimização Adadelta. Na última camada, por se tratar de uma classificação binária, foi utilizada a função de ativação Sigmoid. Todas as camadas FCNN foram seguidas por uma camada de Dropout, com uma taxa de desligamento de $20 \%$

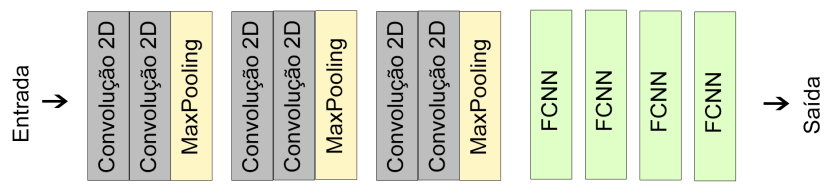

Fig. 8. Arquitetura da Rede Neural Convolutiva desenvolvida. Fonte: autor.

\section{RESUltados E DiscUSS ÃO}

A fim de avaliar o método proposto de classificação, os experimentos realizados foram guiados seguindo o protocolo de Holdout. Neste protocolo, $70 \%$ da base de dados é utilizado como treinamento enquanto os $30 \%$ restantes são utilizados como teste e validação. Desta forma, o modelo de classificação treina em uma base de dados e é avaliado em amostras diferentes dispostas em um subset.

Para comparação e validação do método em relação aos demais trabalhos da literatura, foram calculadas as seguintes unidades métricas: acurácia, AUC e sensibilidade. A AUC é uma métrica largamente utilizada quando existe diferença entre o número de amostras de cada classe contidas na base de dados. De forma mais sensível à acurácia tradicional, esta métrica avalia a eficiência do método como um todo e, portanto, foi a métrica escolhida neste projeto para maximização.

De modo geral, as imagens de fundo de olho, devido aos seus mais variados padrões, representam um grande desafio. As metodologias de Visão Computacional disponíveis na literatura apresentam resultados satisfatórios quanto à classificação destas imagens. A Tabela II contém um comparativo do método proposto com os demais trabalhos apresentados na Seção 2 deste trabalho. A metodologia proposta aqui, comparada ao atual estado da arte em classificação da retinopatia 
diabética, apresentou resultados competitivos de classificação além de fornecer uma sequência de padronização que possa ser utilizada posteriormente em outras metodologias.

TABELA II

COMPARATIVO ENTRE O MÉTODO PROPOSTO E OS DEMAIS MÉTODOS DE CLASSIFICAÇÃO DA RETINOPATIA DIABÉTICA. FONTE AUTOR.

\begin{tabular}{|c|r|c|c|c|}
\hline Autor & ACC & AUC & SEN & ESP \\
\hline Sinthanayothin et al. [22] & - & - & $88 \%$ & $99 \%$ \\
\hline Verma et al. [23] & $90 \%$ & - & - & - \\
\hline Pratt et al. [18] & $75 \%$ & - & - & $95 \%$ \\
\hline Gargeya et al. [12] & - & $97 \%$ & $94 \%$ & $98 \%$ \\
\hline Método Proposto & $\mathbf{8 4 \%}$ & $\mathbf{9 2 \%}$ & $\mathbf{7 9 \%}$ & - \\
\hline
\end{tabular}

\section{CONCLUSÃO}

A diabetes é uma doença considerada epidemia mundial. A retinopatia diabética é uma das principais complicações da diabetes quando não tratada adequadamente. $\mathrm{O}$ desenvolvimento de tecnologias que auxiliam no diagnóstico e acompanhamento desta doença é de considerável importância para evitar o desenvolvimento de cegueira no paciente.

Neste trabalho foi desenvolvida uma metodologia de classificação de imagens de fundo de olho. Dada a grande diversidade de bases de dados e as dificuldades dos classificadores tradicionais em lidar diretamente com essas imagens, uma etapa de processamento de imagens é proposta com intuito de realçar características e padronizar a informação enviada ao classificador. Obteve-se pela atual metodologia, um modelo de classificação com satisfatória eficácia no diagnóstico da retinopatia diabética.

Em trabalhos futuros, pretende-se um desenvolver uma aplicação para o apoio ao diagnóstico que possa ser utilizado, por exemplo, em lugares remotos ou onde há carência nos serviços de atenção secundária. Novas metodologias de classificação e de padronização também podem ser desenvolvidas.

\section{REFERÊNCIAS}

[1] Data Science Academy. Deep learning book, 2019. Disponível em: http://www.deeplearningbook.com.br/. Acesso em: 15/03/2020.

[2] IDF Diabetes Atlas. 9th, 2019.

[3] Kierstan Boyd. Diabetic retinopathy diagnosis, 2019. Disponível em: https://www.aao.org/eye-health/diseases/diabetic-retinopathy-diagnosis. Acesso em: 08/03/2020.

[4] Sociedade Brasileira de Diabetes. Diretrizes da sociedade brasileira de diabetes, 2020. Disponível em: https://www.diabetes.org.br/. Acesso em: 20/03/2020.

[5] Secretaria de Saúde do Estado do Paraná. Linha guia de diabetes mellitus, 2018., 2018.2 Disponível em: http://www.saude.pr.gov.br/arquivos/File/linhaguiadiabetes2018.pdf. Acesso em: 08/03/2020.

[6] Etienne Decenciere, Guy Cazuguel, Xiwei Zhang, Guillaume Thibault, J-C Klein, Fernand Meyer, Beatriz Marcotegui, Gwénolé Quellec, Mathieu Lamard, Ronan Danno, et al. Teleophta: Machine learning and image processing methods for teleophthalmology. Irbm, 34(2):196-203, 2013.

[7] Etienne Decenciere, Xiwei Zhang, Guy Cazuguel, Bruno Lay, Béatrice Cochener, Caroline Trone, Philippe Gain, Richard Ordonez, Pascale Massin, Ali Erginay, et al. Feedback on a publicly distributed image database: the messidor database. Image Analysis \& Stereology, 33(3):231-234, 2014.
[8] BS Divya, Kamalraj Subramaniam, and HR Nanjundaswamy. Human epithelial type-2 cell image classification using an artificial neural network with hybrid descriptors. IETE Journal of Research, 66(1):30$41,2020$.

[9] Hospital dos Olhos. Informação: Retinopatia diabética., 2019. Disponível em: https://www.sadalla.com.br/index/especialidadesoftalmo logia/retina-tratamento-cirurgia/. Acesso em: 08/03/2020.

[10] Frederick L Ferris. How effective are treatments for diabetic retinopathy? Jama, 269(10):1290-1291, 1993

[11] SS Furuie, MA Gutierrez, NB Bertozzo, JCB Figueriedo, and M Yamaguti. Archiving and retrieving long-term cineangiographic images in a pacs. In Computers in Cardiology 1999. Vol. 26 (Cat. No. 99CH37004), pages 435-438. IEEE, 1999.

[12] Rishab Gargeya and Theodore Leng. Automated identification of diabetic retinopathy using deep learning. Ophthalmology, 124(7):962969, 2017.

[13] R Klein. Epidemiology of eye disease in diabetes. Diabetes and Ocular Disease, 2000.

[14] Ronald Klein, Barbara EK Klein, and Scot E Moss. Visual impairment in diabetes. Ophthalmology, 91(1):1-9, 1984.

[15] RS Marques. Segmentação automática das mamas em imagens térmicas. Master's thesis, Instituto de Computação, Universidade Federal Fluminense, Niterói, RJ, Brasil, 2012.

[16] A Nithya, Ahilan Appathurai, N Venkatadri, DR Ramji, and C Anna Palagan. Kidney disease detection and segmentation using artificial neural network and multi-kernel k-means clustering for ultrasound images. Measurement, 149:106952, 2020.

[17] Prasanna Porwal, Samiksha Pachade, Ravi Kamble, Manesh Kokare, Girish Deshmukh, Vivek Sahasrabuddhe, and Fabrice Meriaudeau. Indian diabetic retinopathy image dataset (idrid): A database for diabetic retinopathy screening research. Data, 3(3):25, 2018.

[18] Harry Pratt, Frans Coenen, Deborah M Broadbent, Simon P Harding, and Yalin Zheng. Convolutional neural networks for diabetic retinopathy. Procedia Computer Science, 90:200-205, 2016.

[19] Prachi R Rajarapollu, Debashis Adhikari, and Nutan V Bansode. Use of artificial neural network for abnormality detection in medical images. In Optimization in Machine Learning and Applications, pages 1-12. Springer, 2020.

[20] Poonam M Rokade and Ramesh R Manza. Automatic detection of hard exudates in retinal images using haar wavelet transform. eye, 4(5):402410, 2015.

[21] Karen Simonyan and Andrew Zisserman. Very deep convolutional networks for large-scale image recognition. arXiv preprint arXiv:1409.1556, 2014.

[22] Chanjira Sinthanayothin, James F Boyce, Tom H Williamson, Helen L Cook, Evelyn Mensah, Shantanu Lal, and David Usher. Automated detection of diabetic retinopathy on digital fundus images. Diabetic medicine, 19(2):105-112, 2002.

[23] Kanika Verma, Prakash Deep, and AG Ramakrishnan. Detection and classification of diabetic retinopathy using retinal images. In 2011 Annual IEEE India Conference, pages 1-6. IEEE, 2011.

[24] Guangfen Wei, Gang Li, Jie Zhao, and Aixiang He. Development of a lenet-5 gas identification cnn structure for electronic noses. Sensors, 19(1):217, 2019.

[25] Daniel Welfer, Jacob Scharcanski, and Diane Ruschel Marinho. A coarse-to-fine strategy for automatically detecting exudates in color eye fundus images. computerized medical imaging and graphics, 34(3):228235,2010

[26] Doaa Youssef and Nahed H Solouma. Accurate detection of blood vessels improves the detection of exudates in color fundus images. Computer methods and programs in biomedicine, 108(3):1052-1061, 2012.

[27] Yufeng Zheng, Clifford Yang, and Alex Merkulov. Breast cancer screening using convolutional neural network and follow-up digital mammography. In Computational Imaging III, volume 10669, page 1066905. International Society for Optics and Photonics, 2018.

[28] Karel Zuiderveld. Contrast limited adaptive histogram equalization. In Graphics gems IV, pages 474-485. Academic Press Professional, Inc., 1994. 